\title{
Enterprise Intelligence Platform in the airline industry
}

\author{
G. Dragosavac ${ }^{1}$, A. Viljoen ${ }^{2} \&$ C. Badenhorst ${ }^{3}$ \\ ${ }^{1}$ Analytical Intelligence, SAS Institute, Cape Town, RSA \\ ${ }^{2}$ New Beginnings, Johannesburg, RSA \\ ${ }^{3}$ Business Intelligence, SAS Institute, Johannesburg, RSA
}

\begin{abstract}
If the airline industry could be described in two words, it would be "intensely competitive". The airline industry generates billions of dollars every year and still has a cumulative profit margin of less than $1 \%$. The reason for this lies in this industry's vast complexity. Airlines have a multitude of different business issues that need to be solved at once, such as the globally uneven playing field, revenue vulnerability, an extremely variable planning horizon, high cyclicality and seasonality, fierce competition, excessive government intervention and high fixed and low marginal cost. To ensure the best chance for full economic recovery, airlines should fully leverage their most prolific asset - data. Data used in conjunction with innovative technologies that would allow the creation of an Enterprise Wide Intelligence Platform, will provide the capabilities for a comprehensive intelligent management and decision-making system throughout the enterprise. The ultimate benefits of implementing and using an enterprise wide intelligence platform, together with airline business acumen and experience would include timely responses to current and future market demands, better planning and strategically aligned decision making, and clear understanding and monitoring of all key performance drivers relevant to the airline industry.

Achieving these benefits in a timely and intelligent manner will ultimately result in lower operating costs, better customer service, market leading competitiveness and increased profit margin and shareholder value. This paper demonstrates the vision and benefits of establishing an Enterprise Wide Intelligence Platform for the airline industry, based on a leading airline proof of concept case study.
\end{abstract}

Keywords: Enterprise Intelligence Platform for airlines, solution for airline industry. 


\section{Introduction}

Airlines throughout the world are currently facing an unprecedented financial crisis. Factors contributing to this crisis are low customer satisfaction, overtraded markets, insufficient and under utilization of aircraft capacity, poor labor relations, excessive government intervention, high labor costs, ever increasing oil prices resulting in spiraling fuel costs, and generally high operational costs. The low profit to turnover ratio of airlines has been further exacerbated by growing low-fare competition, increasing security costs, and frequent dynamic shifts in air travel consumer behavior. The historical business model of many network airlines now appears to be unable to support sustained profitability under any but the most favorable economic conditions. The industry is at a turning point. The market dictates an "adapt or die" policy, and the airlines that whish to survive will face the challenge of having to make significant changes to their current archaic business model. To do this requires far more allowance for innovative technologies that would allow airlines to build an end-to-end Enterprise Wide Intelligence Platform. The core capabilities of these technologies will ensure the flow of consistent, repeatable and reliable enterprise wide intelligence needed to tackle all the challenges the industry is facing.

\section{Conceptual overview of Enterprise Intelligence Platform for airlines}

\subsection{What is the Enterprise Intelligence Platform}

Technology that helps to define, align and drive key strategies by providing a comprehensive framework for supporting business specific needs across the different business units of an airline company is termed an Enterprise Intelligent Platform (EIP). The prudent question to ask here is; how would an airline know if they have such platform in place already? The answer is simply that a true EIP requires pre-integrated technology capabilities that would enable airlines to control the process of turning normal dead data into intelligence. Airlines utilise several large and inflexible "legacy" IT systems to provide its functional needs (e.g. reservations, loyalty programs, technical, cargo, etc.) The EIP runs on airline operational data which goes through a process of being integrated, enriched, cleansed, quality checked, optimally stored and analysed. As a result of all these activities, new intelligence is derived from data information, and delivered to the intelligence users, empowering them to intelligent decision making across the enterprise. As a result potentially useful, non-trivial and actionable intelligence is surfaced that delivers new insights, provides the answers, drives strategy and addresses the core business issues and not just the peripheral symptoms.

It is this synergistic integrated approach that makes the difference between general, isolated pockets of information and intelligent consistent, usable enterprise wide effectiveness that the airline industry needs in order to survive in a complex and demanding world. 


\subsection{Purpose of the Enterprise Intelligence Platform for airlines}

Purpose of an EIP for airlines is to bridge what is called the Information-tointelligence gap. The disparity between what an airlines has - which is prolific amounts of data from disparate source systems - and what an airline wants which is to achieve strategy alignment for a competitive edge; whether it be through compliance, increased profitability, decreased risk, or to better manage performance, planning, etc

\subsection{Components of Enterprise Intelligence Platform}

Figure 1 below depicts the five major components the EIP, and each one will be discussed in turn in the next couple of paragraphs.

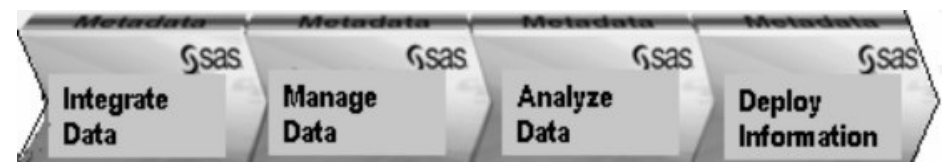

Figure 1: $\quad$ EIP components.

\subsubsection{Metadata}

The centrally shared Metadata forms the backbone of the EIP and contains descriptive information about all technical and business related items included into the Enterprise Business Intelligence platform. Metadata allows:

- One single point of administrative control and maintenance for the whole enterprise business intelligence environment,

- Integrated Authentication and Authorization,

- Ensures one single copy of the data and metadata truth for all intelligence users across the enterprise.

\subsubsection{Data integration}

Data integration involves collecting, cleaning, quality checking, integrating and enhancing data. This is where data extraction, transformation and loading (ETL) happen, as well as transforming unstructured data (emails, voicemails, written notes, etc.) as well as correcting inconsistencies (data quality).

\subsubsection{Data management}

Data Management involves the process of optimized intelligent storage of the data in synchronized repositories from which information can be extracted and intelligence applied to solve a specific strategic business issue.

\subsubsection{Data analysis}

Data Analysis is simply deriving intelligence of insight from information by looking at it in different ways. Some insights come from simple data management tasks such as making lists and ranking data, etc. These tasks are standard functionality of query and reporting capabilities. Other insights come 
from using statistical analysis, forecasting, optimization and data mining. These advanced analytics would help the airline companies to eliminate the guesswork from decisions to gain a competitive edge based on cold hard factual intelligence which makes everyone happier - from the corporate shareholders to regulators, employees and customers.

\subsubsection{Intelligence deployment}

Data Integration, Data Staging, and Data Analysis are all critical preceding steps to successful intelligence deployment. Intelligence Deployment is the process of delivering the right information to the right people at the right time, and in a format they are familiar with to ensure they are empowered to make the most knowledgeable intelligence decisions possible. The deployment of corporate intelligence - has many facets. It could be a report to a manager, a triggering of an email to unhappy customers, a mapping of metrics to a Scorecard or to a regulatory compliance report. Another facet is how quickly and accurately intelligence can be applied to a business problem or an emerging opportunity. For example, if an airline can identify the most profitable of its customers they can get to them first, or if you can find those who are most likely to defraud you before they actually do, you can take immediate and intelligence based measures to protect yourself.

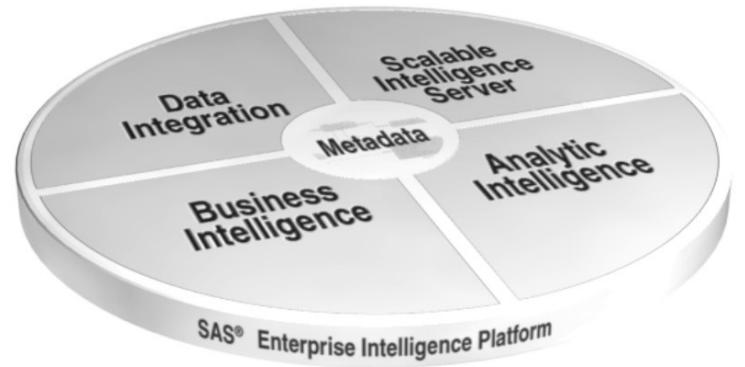

Figure 2: $\quad$ The Integrated EIP.

\subsection{The Pre-Integrated Enterprise Intelligence Platform}

There are three levels of integration that needs to take place within an enterprise to support business intelligence:

- Architecture Integration - the EIP needs to be flexible enough to integrate with the current existing enterprise architecture infrastructure.

- Data Integration - the EIP needs to be flexible and able enough to access data or information from any existing disparate electronic raw data source

- System Integration - all the components of the EIP needs to have a synergy as a pre-integrated whole to ensure consistent, reliable, repeatable and quality intelligence throughout the enterprise. 
The EIP for airlines fully supports all levels of required integration as standard. Not only is the information interchangeable across the enterprise, the process is seamless, efficient and rapid. The result is a faster "time to intelligence" - delivering business-critical answers to the whole enterprise in a timely manner. This gives an airline more time to react to changing markets (e.g. ability to bring products to market faster, identify and target your most valuable customers, improve service quality, and better manage shareholder expectations).
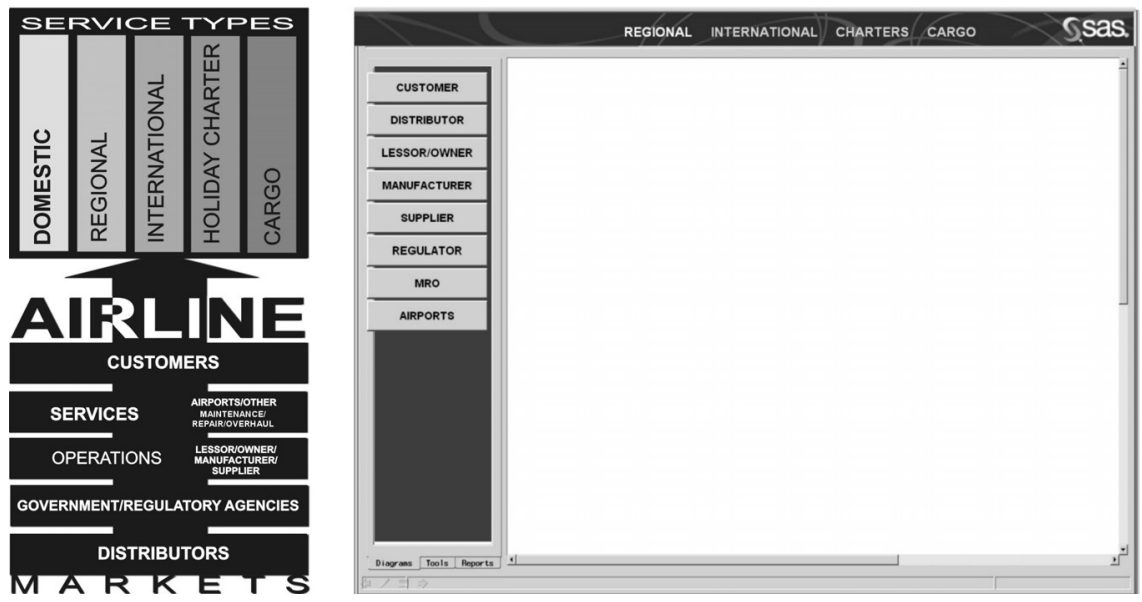

Figure 3: Airline industry business model scheme (left) and an example of EIP Portal interface reflecting and supporting the industry business model (right).

\section{Addressing the business issues}

\subsection{A business model for the in airline industry}

Key to successful deployment of technological advances in airline industry is to be able to anticipate how the current business model will change to survive in tough market conditions.

\subsection{Examples of how an Enterprise Intelligent Platform would address some of the business issues arising from the new airline business model}

Some of the challenges that can be successfully addressed by EIP are:

- The need for accurate daily and weekly performance measurement reports (e.g. "flash/estimated" revenue, operating costs and net contribution reports for every aircraft's actual flight per sector/route).

- The need to better manage all aspects of risk.

- The need for better impact analysis and more effective optimization of all resources as well as being able to produce accurate passengerrevenue forecasts, 
- The need for a holistic, $360^{\circ}$ view of the airline industries customers, suppliers, service providers and distributors.

- The need for expense verification models in order to better control all industry cost aspects.

\subsubsection{Issues related to performance measurements}

Airlines usually operate in a globally competitive environment and therefore require prompt and accurate enterprise performance measurements. Furthermore, airlines are volume driven and small variations (passengers flown, fuel spent/bought, load carried) can multiply into major effects - therefore appropriate and timely action is critical. Airlines suffer substantial difficulties to produce daily/weekly reliable performance measurements. Current airlines "legacy" IT systems such as Revenue Accounting, require several weeks after a month end to generate revenue results for every flight per sector/route. EIP can automate production of daily activity reports such as number of passenger flown per flight/sector, distance flown, etc which can be used to provide estimated performance measurements such as daily or weekly revenues for specific routes or sectors.

Figure 4 depicts the Portal front-end of the EIP with an example of two reports - "Passenger Revenue Report" and "Net-Margin Analysis" report. These and other similar reports are generated in real time by the press of a button. What is hidden away from the business user is the complex back-end architecture and processes that collects the information, cleans it, and puts it in a appropriate format, does all necessary calculations and then displays it in the preferred format as required and understood by a business user to enable appropriate action.

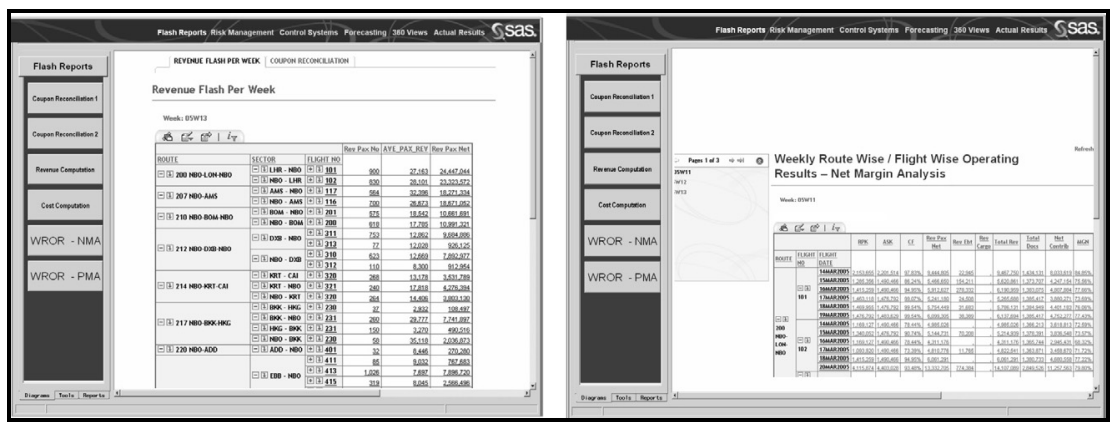

Figure 4: Example of predefined Performance Measurement reports generated automatically on user's request.

\subsubsection{Issues related to risk management}

The global airline industry has been subjected to major catastrophes over the past years. It is accordingly imperative for airlines to develop various risk management models and strategies to protect themselves from negative impact of these types of events. Furthermore, due to the global playing field, airlines often earn its revenues and pay its costs in different baskets of currencies (e.g. 
USD, Euro, GBP etc). As a result there is frequently a mismatch between the flow of revenue receipts and expenses of each basket of currency - creating risk exposure. Using Enterprise Intelligence Infrastructure, relevant data can be gathered, consolidated and cleaned, risk can be modeled, and risk exposure can be measured and presented on "as and when" basis, as requested by business user.

\subsubsection{Issues related to control and verification}

Airline carriers require a number of control and verification models to be able to control costs arising from its various operational activities. To enable this, airlines have a pressing need for a complete and integrated repository of flight information data gathered from all its disparate business units. This will enable computation of various efficiency analytics - e.g. planed fuel usage compared with actual fuel usage per aircraft, crew utilization (roster optimization). These issues could also be fully addressed by the EIP, which will access, consolidate and analyze relevant flight and aircraft data. In turn this would help to create a $360^{\circ}$ view of each flight and aircraft, allowing the business users to dramatically improve their control and verification systems. Figure 5 depicts an example contribution and verification report.

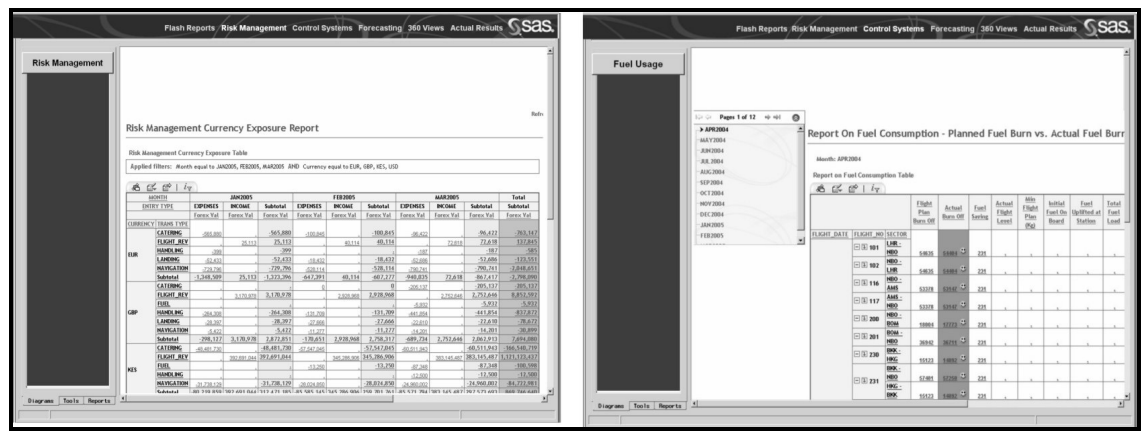

Figure 5: Example of risk management and control and verification reports.

\subsubsection{Issues related to be able to better forecast}

Airlines require the development of an effective and holistic forecasting model to regularly assess the impact of options and alternatives such as increasing aircraft seats available, adjusting fares, introducing new routes etc. Forecasts should also take account of actual statistical trends and results e.g. actual passengers carried and actual average fares earned. Such forecasts should then be compared against budgets and prior year performance. The EIP has a market leading and powerful forecasting engine capable of generating large number of forecasts automatically and making them available to the people who would used them for sound decision making. 


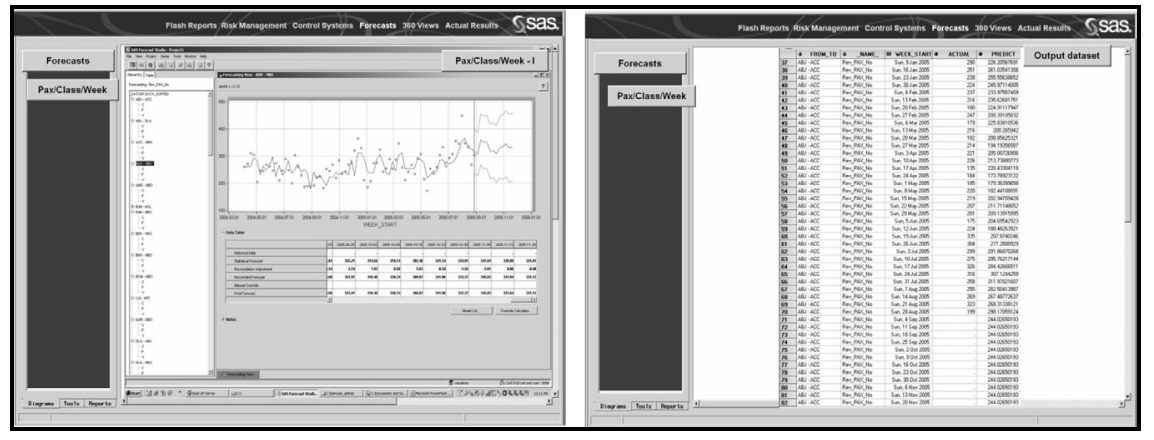

Figure 6: Example of forecasting output.

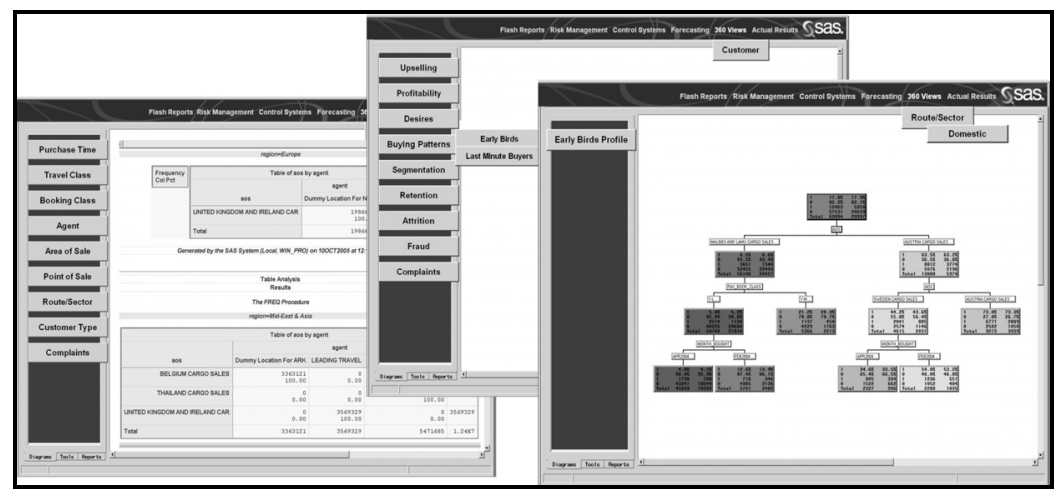

Figure 7: Example results of complex analytics that can be processed and surfaced by the EIP Portal.

\subsubsection{Issues related to a lack of a holistic view of core business components}

Airlines would greatly benefit from knowing and understanding its business environment along some of the key business issues, such as performance, behavior, risk, profitability, etc. Using customers as an example - the main objective would be to enrich the knowledge about individual customers leading to new strategic customer segments. This intelligence would allow airlines to reap the host of benefits such as successful, targeted customer promotions, crossselling and up-selling campaigns for different flights and booking classes leading to improved yield and revenue. For example, it would give airlines the power of knowing to limit discounts on flight routes which are usually over-booked, allowing the large number of passengers to compete for high profit seats immediately prior to departure. Such multidimensional views of the business can help the airline to better serve its customers through more effective, efficient and personalized service, receiving in return customer loyalty, support and market share, all leading to higher profitability. 


\section{Conclusion}

The EIP for airlines is designed on usable, interoperable, scalable, and manageable technology, and encompasses all aspects of turning information into strategically aligned, powerful and accurate intelligence and empowering the business user into intelligent action by ensuring the delivery of the right intelligence to the right business user in the right format in a timely manner. The EIP is built on core technological components of data integration, data management, data analysis and information deployment, all of these components being fed by centrally shared enterprise wide metadata. Built into these core technology components are airline specific data models, statistical and analytical models, pre-written reports and all necessary training and methodologies for a successful and sustainable EIP for airlines implementation. All of these items collectively give the EIP the capability and capacity to address the host of the burning issues prevalent in the airline industry.

\section{References}

[1] Lisa Pritscher, Hans Feyen, Data Mining and Strategic Marketing in the Airline Industry, Conference Paper.

[2] Tom Hansson, Dr. Jurgen Ringbeck, Dr Markus Franke Airlines: New Operating Model, Article.

[3] A.T. Kearney, The Society Of British Aerospace Companies, The Emerging Airline Industry, Joint Study.

[4] Nawal Taneja, Airline Survival Kit, Book.

[5] SAS Institute, The SAS Enterprise Intelligence Platform, SAS Analytic Intelligence, White Paper. 